\title{
Synthesis of a reusable oxotungsten-containing SBA-15 mesoporous catalyst for the organic solvent-free conversion of cyclohexene to adipic acid
}

\author{
Ching-Yuan Cheng a, Kuan-Jiuh Lin ${ }^{\text {a,*, }}$, Muppa R. Prasad a, Shu-Juan Fu a, \\ Sheng-Yueh Chang ${ }^{a}$, Shin-Guang Shyu ${ }^{b}$, Hwo-Shuen Sheu ${ }^{c}$, Chia-Hao Chen ${ }^{c}$, \\ Cheng-Hao Chuang ${ }^{d}$, Minn-Tsong Lin ${ }^{d}$ \\ a Department of Chemistry, Center of Nanoscience and Nanotechnology, National Chung-Hsing University, 250, Kuo Kuang Road, \\ Taichung 402, Taiwan, ROC \\ ${ }^{\mathrm{b}}$ Institute of Chemistry, Academia Sinica, Taipei, Taiwan \\ ${ }^{\mathrm{c}}$ National Synchrotron Radiation Research Center, Hsinchu 300, Taiwan \\ d Department of Physics, National Taiwan University, Taipei 106, Taiwan and Institute of Atomic and Molecular Sciences, \\ Academia Sinica, Taipei 106, Taiwan
}

Received 8 March 2006; received in revised form 18 October 2006; accepted 18 October 2006 Available online 2 November 2006

\begin{abstract}
An oxotungsten-silica mesoporous structure (WSBA-15) has a hierarchical crystalline architecture in which the W dopants possess tetrahedral coordination geometries for the mixed-valence states $\mathrm{W}^{6+}, \mathrm{W}^{5+}$, and $\mathrm{W}^{4+}$. The WSBA-15 catalyst can be recycled-without any loss of activity - for the direct oxidation $\left(30 \% \mathrm{H}_{2} \mathrm{O}_{2}\right)$ of cyclohexene to colorless, crystalline adipic acid (55\% yield) under organic solvent-free conditions.
\end{abstract}

(C) 2006 Elsevier B.V. All rights reserved.

Keywords: SBA15; Mesoporous; Oxotungsten; Cyclohexene; Adipic acid; Hydrogen peroxide; TEM; Raman; UV-Vis; Powder X-ray diffraction; XPS

\section{Introduction}

Periodic mesoporous silica (PMS) samples possessing crystal-like wall structures have a very promising range of future applications. The development of PMS materials, which include mesostructured MCM-41 [1,2], HMS [3,4], and SBA-15 $[5,6]$ molecular sieves, has opened up a vast field of research with respect to both their structural properties and their many technological applications (e.g., as molecular sieves, catalysts, and sensors and in nanoelectronics [7-10]). In the field of catalysis, these silica materials offer new opportunities for creating highly disperse and

\footnotetext{
${ }^{*}$ Corresponding author. Tel.: +886 4 22840411x802; fax: +886 2 22862547.

E-mail address: kjlin@dragon.nchu.edu.tw (K.-J. Lin).
}

more-accessible catalytic sites in which metal ions are grafted onto the internal surfaces of the pores [11-14]. Tungsten-containing silica nanostructures have been attracting considerable attention recently because of their remarkable activities as catalysts $[15,16]$. In addition, the idea by applying tungsten species as catalyst was since 1998, Noyori et al. have used sodium tungstate $\left(\mathrm{Na}_{2} \mathrm{WO}_{4}\right)$ as a catalyst to convert cyclohexene to adipic acid in the homogeneous condition, which achieved about $78 \%$ yield [17]. However, in the heterogeneous catalysis, a great amount of effort has been exerted in the past five years to improve the dispersion of low-condensed tungsten within mesoporous silicas and to increase selectivity and yield of product [18-21]; unfortunately, tests of these materials' catalytic properties, when reported, have indicated that only a small number of the redox and/or acid sites are active, i.e., 
turnover numbers are very low. For the development of tungsten-containing PMS materials, it is extremely desirable to dope tetrahedral $\left\{\mathrm{WO}_{4}\right\}$ species into silica frameworks that have a very high degree of cross-linked silicate tetrahedra $\left\{\mathrm{SiO}_{4}\right\}$. In this paper, we report a PMS system based on a mixed-valence oxotungsten-silica mesoporous structure (WSBA-15) incorporating tetrahedral tungsten units. This system offers great promise for application as a reusable catalyst for the oxidation of cyclohexene to adipic acid.

\section{Experimental section}

\subsection{Synthesis of WSBA-15}

Solution A: $\mathrm{Na}_{2} \mathrm{WO}_{4}(0.130 \mathrm{~g}, 0.41 \mathrm{mmol})$ was dissolved in $30 \%$ aqueous $\mathrm{H}_{2} \mathrm{O}_{2}(0.5 \mathrm{~mL})$. Solution B: Pluronic 123 $(4.00 \mathrm{~g})$ was dissolved with $2 \mathrm{M} \mathrm{HCl}(120 \mathrm{~g})$ in $\mathrm{H}_{2} \mathrm{O}$ $(30 \mathrm{~mL})$. Tetraethylorthosilicate (TEOS, $8.50 \mathrm{~g}$ ) and Solution A were introduced simultaneously into Solution B under vigorous stirring at $35^{\circ} \mathrm{C}$ for $20 \mathrm{~h}$. After leaving the resulting gel to age at $80^{\circ} \mathrm{C}$ overnight without stirring, the yellow solid product was filtered, washed with distilled water, and then air dried at room temperature. The as-synthesized WSBA- 15 product was calcined at $500{ }^{\circ} \mathrm{C}$ for $6 \mathrm{~h}$ and then treated with $1 \mathrm{M}$ ammonium acetate at $80^{\circ} \mathrm{C}$ for $6 \mathrm{~h}$. The washed WSBA-15 was colorless. ICP mass spectrometric analysis indicated that the $\mathrm{Si} / \mathrm{W}$ molar ratio of the washed WSBA-15 was 5300 based, suggesting that the large amounts of loosely bonded tungsten species were readily removed when using ammonium acetate solution. Moreover, the complete replacement of $\mathrm{Na}^{+}$by $\mathrm{NH}_{4}^{+}$ions was readily accomplished, as confirmed through energydispersive X-ray fluorescence analysis.

\subsection{Catalyst reaction}

The catalytic reactions were performed in batch mode using a glass vessel fitted with a condenser circulating chilled water. The vessel was heated in an oil bath to $85^{\circ} \mathrm{C}$. The substrates [cyclohexene $(1.00 \mathrm{~g})$ and $30 \%$ aqueous $\mathrm{H}_{2} \mathrm{O}_{2}(5.5 \mathrm{~mL})$ ] and catalyst [washed WSBA-15 $(0.20 \mathrm{~g})]$ were introduced into the glass vessel and the contents were stirred using a magnetic stirrer. The biphasic reaction mixture was heated subsequently at $85^{\circ} \mathrm{C}$ for either 13 or $30 \mathrm{~h}$ at a stirring rate of $1000 \mathrm{rpm}$. Small amounts of the reaction medium were collected from the filtrate of the catalytic reaction and then analyzed by gas chromatography (Hewlett 6890 Packard series gas chromatograph) using an HP-5 capillary column; the products were confirmed through analysis using a Hewlett 5973 Packard mass-selective detector. Trimethylsulphonium hydroxide was added to the samples to form esters from any acids; the samples were then injected into the gas chromatograph. The products appeared in the GC traces in the following sequence: cyclohexene epoxide, cyclohexanedione, cyclohexanone-2-ol, cyclohexanediol, dimethyladi- pate. The collection and identification of adipic acid was performed by adding methanol and $\mathrm{H}_{2} \mathrm{O}$ to filter the reaction medium and wash the catalyst. The solvents of the collected mixture were removed under rotary evaporation and the solid was recrystallized from EtOAc to provide adipic acid, which was identified by ${ }^{1} \mathrm{H}$ NMR spectroscopy. The recovered catalyst was air dried and calcined at $450{ }^{\circ} \mathrm{C}$ for $6 \mathrm{~h}$ prior to use in further catalytic cycles.

\subsection{Characterization}

High-resolution transmission electron microscopy (HRTEM) measurements were performed using a $220 \mathrm{kV}$ JEOL TEM microscope. The samples were obtained from a $0.01 \%$ solution in ethanol and measured on carboncoated copper grids. The Raman spectra of the solid materials were recorded at room temperature by focusing the laser spot with a Nanofinder@30 (Tokyo Instruments, Inc) spectrometer and using the excitation line of $488 \mathrm{~nm}$ for a semiconductor laser and $633 \mathrm{~nm}$ for a $\mathrm{He}-\mathrm{Ne}$ laser (power of laser spot: $1.5 \mathrm{~mW} / \mathrm{cm}^{2}$; resolution: $4 \mathrm{~cm}^{-1}$ ); the sample was placed on a quartz substrate. UV-Vis spectra were recorded using a Perkin-Elmer Lambda 900 UV/ Vis spectrophotometer; the solid samples were placed in the reflectance module. Powder X-ray diffraction patterns were collected at the BL-17A Beamline and XPS analysis was performed using the SPEM station equipped with a 16channel energy analyzer of the National Synchrotron Radiation Research Center (NSRRC), Taiwan.

\section{Results and discussion}

Fig. 1 illustrates the methodology we used to develop an organic solvent-free route to the preparation of adipic acid over WSBA-15. Typically, we synthesized WSBA-15 in an acidic medium through the spontaneous self-assembly of

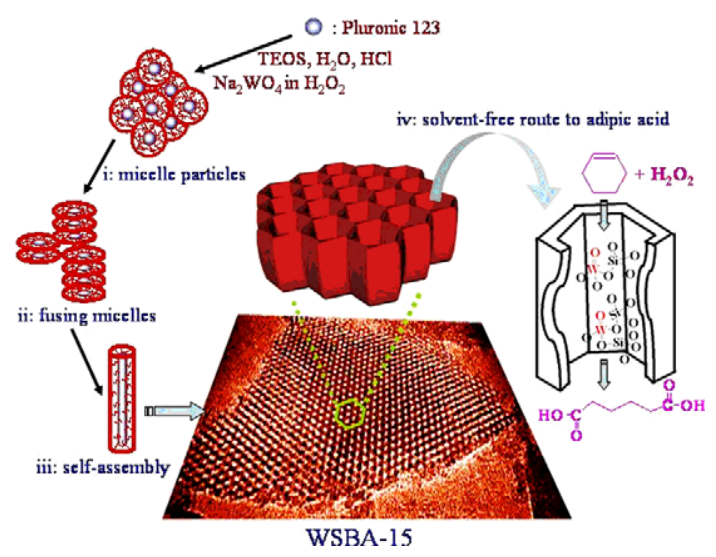

Fig. 1. Conceptual scheme for (i-iii) the synthesis of periodically ordered WSBA-15 mesopores and (iv) organic solvent-free oxidation of cyclohexene to adipic acid in aqueous $\mathrm{H}_{2} \mathrm{O}_{2}$. Incorporating catalytic oxotungsten tetrahedra centers into the framework maintains the hierarchical structure of the mesopores and improves the lifetime and reusability of the heterogeneous catalysts for single-step alkene oxidations. 
silica species in the presence of the structure-directing species, namely poly(ethylene oxide)- $b$-poly(propylene oxide)$b$-poly(ethylene oxide) triblock copolymer (Pluronic 123). To achieve a high dispersion of $\mathrm{WO}_{4}$ species onto the silica framework, we dissolved $\mathrm{Na}_{2} \mathrm{WO}_{4}$ in $\mathrm{H}_{2} \mathrm{O}_{2}$ prior to adding it to the silica precursors of the sol-gel process. The copolymer was removed by calcination at $500{ }^{\circ} \mathrm{C}$ for $6 \mathrm{~h}$. We then treated the calcined WSBA-15 sample with $1 \mathrm{M}$ aqueous ammonium acetate at $80^{\circ} \mathrm{C}$ for $6 \mathrm{~h}$ to remove any loosely bound tungsten species; we also repeated this procedure prior to any further oxidation reactions. We obtained the WSBA- 15 products in a highly reproducible manner (yields $>95 \%$ ) and characterized their ordered, robust mesostructures from X-ray diffraction (XRD), high-resolution transmission electron microscopy (HRTEM). We confirmed the nature of the tungsten species within WSBA-15 by using ICP-MS, UV-Vis diffuse reflection spectroscopy, Raman microscopy, and X-ray photoelectron spectroscopy (XPS).

The XRD pattern for the WSBA-15 mesostructure (Fig. 2a) displays three peaks in the small-angle scattering regime $\left(2 \theta<4{ }^{\circ} \mathrm{C}\right)$ that are indexed as $(100),(110)$, and (200) reflections associated with $p 6 \mathrm{~mm}$ hexagonal symmetry. The intense (100) peak reflects a $d$ spacing of $10.3 \mathrm{~nm}$, which corresponds to a large unit cell parameter $a=12.7 \mathrm{~nm}[a=d(100) \times 2 / \sqrt{ } 3]$. The TEM images of WSBA-15 provide additional structural information; they display the presence of well-ordered hexagonal arrays of one-dimensional channels having uniform size and further confirm that the WSBA-15 samples have a 2D hexagonal structure (Fig. 2b,c). From the high-contrast dark regions in the TEM image of this sample, we estimate that the distance between the mesopores is ca. $12.0 \mathrm{~nm}$ - a value that is in agreement with the value determined from the XRD analysis - and that the silica wall thickness and the pore sizes are 5.5 and $6.5 \mathrm{~nm}$, respectively.

The WSAB-15 sample was colorless, which indicates the absence of any colored crystalline $\mathrm{WO}_{3}$ species on the outside of the framework. The synchrotron radiation XRD analysis also indicated that no significant diffraction patterns corresponding to crystalline $\mathrm{WO}_{3}$ appeared during the course of calcinations up to $500{ }^{\circ} \mathrm{C}$. This result is supported by a comparison of the diffuse reflectances of WSBA-15 and SBA-15 in the UV-Vis region (Fig. 3). We assign unambiguously the broad absorption band near $256 \mathrm{~nm}$ to the (Laporte allowed; $\mathrm{sp}^{3} \rightarrow \mathrm{sd}^{3}$ ) ligand-to-metal electron transition, which is consistent with tetrahedral sites on silica-based materials. The more-intense absorption band observed for WSBA-15 supports our assumption that the majority of the tungsten atoms remain in tetrahedrally coordinated geometries within the SBA-15 framework [22]. We used XPS to provide evidence for the oxidation states of the tungsten atoms in WSBA-15 (Fig. 4a). Our curve-fitting of the W $4 f$ photoelectron spectra suggests that the $\mathrm{W}$ atoms are present in the mixedvalence states $+6(30 \%),+5(47 \%)$, and $+4(23 \%)$. Moreover, a preliminary X-ray absorption near-edge spectrum (XANES) revealed that WSBA-15 has a significant pre-

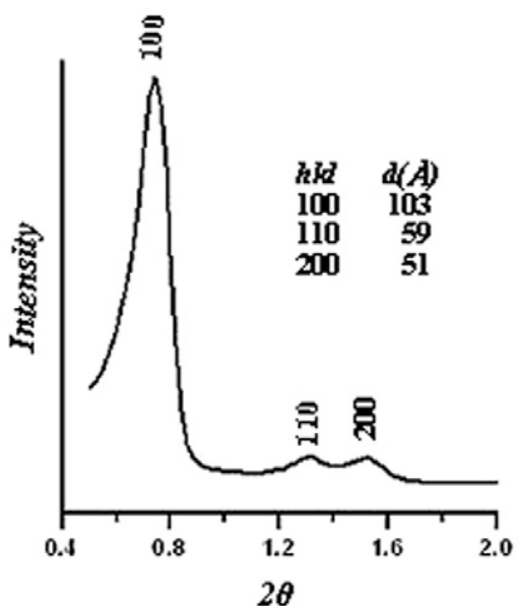

a

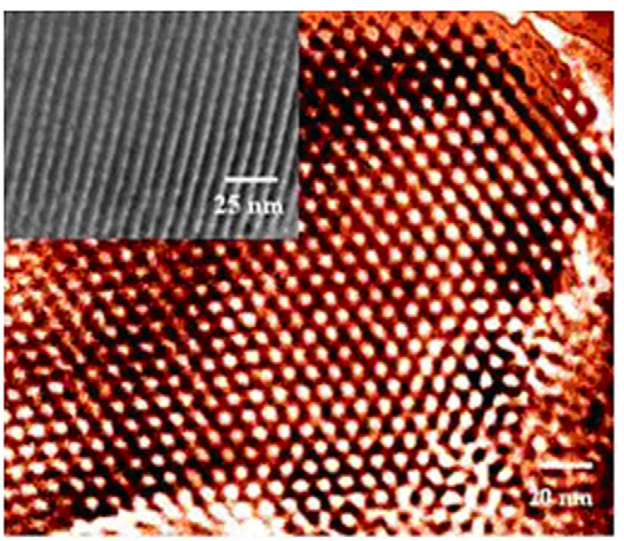

b

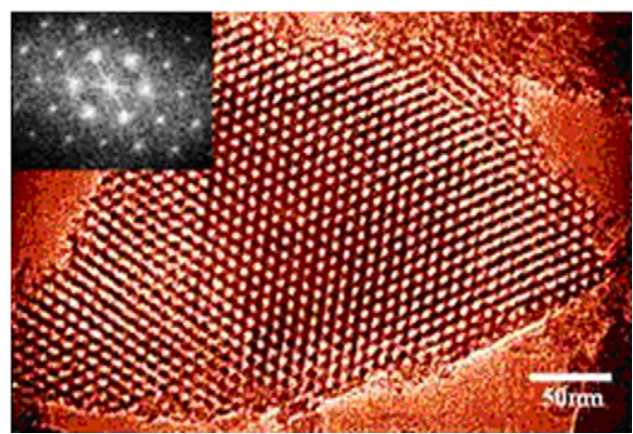

Fig. 2. (a) Low-angle X-ray diffraction pattern for WSBA-15 (synchrotron radiation, $\lambda=1.32633 \AA$ ). (b) HRTEM images of WSBA-15, displaying a well-ordered hexagonal array and the one-dimensional channel structure (inset). (c) HRTEM images and the electron diffraction pattern (inset) of WSBA-15 after a third catalytic oxidation of cyclohexene, recorded with [ 00101$]$ incidence parallel to the channels. Note that the electron diffraction pattern clearly displays high-degree, long-range hexagonal symmetry.

edge at $12103 \mathrm{eV}$ and, thus, it is reasonable to assume that the $\mathrm{W}$ atoms possess tetrahedral coordination geometries [23]. We also verified the structural effect by using Raman microscopy (Fig. 4b). In general, crystalline $\mathrm{WO}_{3}$ is a very strong Raman scatterer; thus, it appears that the broad features offer conclusive evidence for the near-homogeneous distribution of the $\left\{\mathrm{WO}_{4}\right\}$ dopant, similar to that observed for WMCM-41 [19]. Therefore, we tentatively assign the two bands at ca. 988 and $824 \mathrm{~cm}^{-1}$ to the symmetric A1 


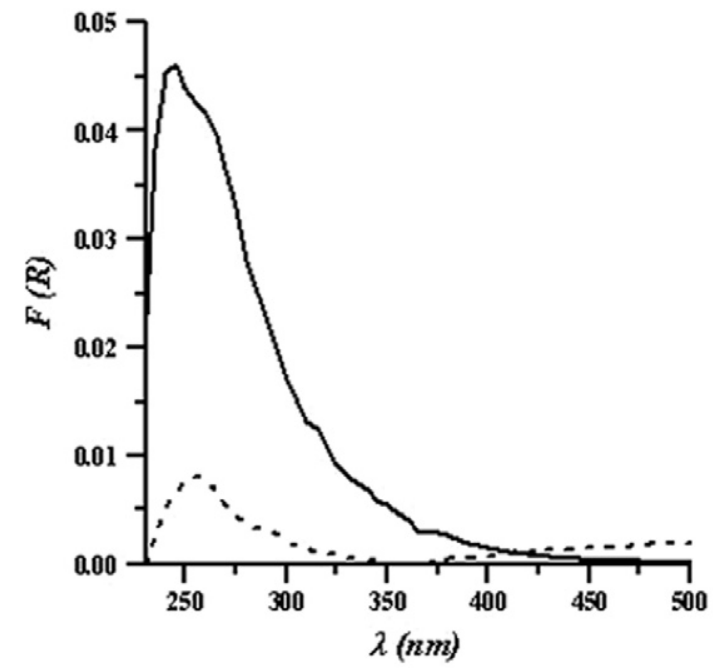

Fig. 3. UV-Vis diffuse reflectance spectra of WSBA-15 ( -$)$ and SBA-15 (- - ) that reflect the near-homogeneous distribution of $\left\{\mathrm{WO}_{4}\right\}$ dopants within the silica framework. The relative reflectance of a powder, $F(R)$, is given by the Kubelka-Munk equation $\left[F(R)=(1-R)^{2} / 2 R\right.$, where $R$ is the ratio of the reflected intensities of the sample and a nonabsorbing standard].

and antisymmetric $\mathrm{F} 2$ stretching modes, respectively, of the $\left\{\mathrm{WO}_{4}\right\}$ structure [24]. In addition, we assign the peak at $988 \mathrm{~cm}^{-1}$ to tungsten "defect" sites possessing terminal oxygen atoms, i.e., vibrations of oxotungsten $(\mathrm{W}=\mathrm{O})$ units on the surface walls of WSBA-15. This value is smaller than those observed under anhydrous conditions for $\mathrm{W}=\mathrm{O}$ groups in tungsten-containing mixed oxides (typically $1015-1030 \mathrm{~cm}^{-1}$ ) [25]. Therefore, we believe that the longer $\mathrm{W}=\mathrm{O}$ bond within WSBA-15 might be indicative of weak interactions between the dangling $\mathrm{W}=\mathrm{O}$ units and water molecules, which suggests that they may provide active centers for the catalytic oxidation of alkenes. Because of the small amount of $\mathrm{W}$ present in WSBA-15 $(\mathrm{Si} / \mathrm{W}=$ ca. 5300$)$, we suggest that $\mathrm{W}$ may be doped with Si. Such doping creates mixed-valence states and oxotungsten tetrahedral structures (Fig. 4c). Moreover, the walls of WSBA-15 are denser than those of other related SBA structures because $\mathrm{W}$ atoms are heavier than $\mathrm{Si}$ atoms and the $\mathrm{WO}_{4}$ tetrahedra are more tightly packed than the
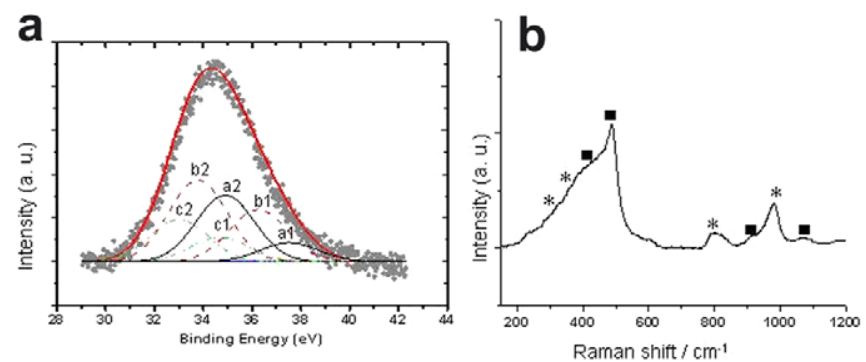

C
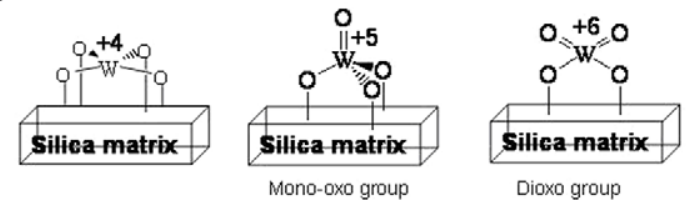

Fig. 4. (a) Synchrotron radiation (photon energy: $369 \mathrm{eV)} \mathrm{XPS} \mathrm{spectra} \mathrm{of}$ WSBA-15: a1 and a2 are components of the $\mathrm{W}^{6+} 4 f$ spectrum, b1 and b2 are components of the $\mathrm{W}^{5+} 4 f$ spectrum; $\mathrm{c}_{1}$ and $\mathrm{c}_{2}$ are components of the $\mathrm{W}^{4+} 4 f$ spectrum. (b) Micro-Raman spectrum of WSBA-15 exhibiting four fundamental vibration modes $\left(^{*}\right)$ of tetrahedral $\left\{\mathrm{WO}_{4}\right\}$ symmetry at ca. $988\left(A_{1}\right), 824\left(F_{2}\right), 373\left(F_{2}\right)$, and $310\left(F_{2}\right) \mathrm{cm}^{-1}$ : the other modes $(\boldsymbol{\square})$ are attributed to silica. (c) Schematic illustration of the tetrahedral structures of mixed-valence oxotungstates.

more-flexible vertex-shared $\mathrm{SiO}_{4}$ tetrahedra. From the results of parallel XRD and thermogravimetric analyses, we found that WSBA-15 can be calcined at up to $1000{ }^{\circ} \mathrm{C}$ in air without collapsing the pore structure and that the mesoscale periodicity is completely preserved after boiling the material in water for $144 \mathrm{~h}$. These findings provide good evidence that WSBA-15 is a hierarchically ordered porous material in which the embedding tungsten-silica matrix provides enhanced chemical, mechanical, and thermal robustness.

The development of "green" practical procedures for the oxidation of six-carbon feedstock is highly desirable - particularly for the medium- and large-scale syntheses of adipic acid, which is an important chemical for the manufacture of nylon-66. Table 1 lists the catalytic performance, including turnover frequencies (TOFs), of WSBA-15 toward the organic solvent-free synthesis of adipic acid. It is noteworthy that the oxidation conversion of cyclohexene reached essentially $100 \%$ after $13 \mathrm{~h}$, and the selectivity toward adipic

Table 1

Oxidation of cyclohexene to adipic acid (AA) under WSBA-15 heterogeneous catalysts

\begin{tabular}{|c|c|c|c|c|c|c|c|}
\hline \multirow[t]{2}{*}{ Catalyst } & \multirow[t]{2}{*}{ Weight (g) } & \multirow[t]{2}{*}{ Time (h) } & \multirow[t]{2}{*}{ Conversion $^{\mathrm{a}}(\%)$} & \multirow[t]{2}{*}{$\mathrm{TOF}^{\mathrm{b}}$} & \multicolumn{3}{|c|}{ Product selectivity (\%) } \\
\hline & & & & & Others $^{\mathrm{c}}$ & Diol & $\overline{\mathrm{AA}}$ \\
\hline WSBA-15 & 0.20 & 13 & 100 & 404 & 10.5 & 59.5 & 30.0 \\
\hline WSBA- $15^{\mathrm{d}}$ & 0.20 & 30 & 100 & 354 & 11.3 & 42.8 & 45.9 \\
\hline TiAPO-5 [13] & 0.50 & 24 & 50 & 20 & 22.5 & 64.4 & 13.1 \\
\hline TiAPO-5 [13] & 0.50 & 72 & 100 & 105 & 39.7 & 30.0 & 30.3 \\
\hline WMCM-41 [16] & 0.13 & 24 & $98^{\mathrm{e}}$ & 63 & & & - \\
\hline
\end{tabular}

${ }^{\text {a }}$ Conversion was determined using GC-MS analysis.

${ }^{\mathrm{b}}$ TOF $=$ moles of adipic acid produced per mole of metal $(\mathrm{W})$ per hour (WSBA- 15 contains $5.8 \times 10^{-2} \mathrm{wt} \% \mathrm{~W}$, as determined by ICP-MS).

${ }^{c}$ The other products included 2-cyclohexene-ol, 2-cyclohexenone, 2-hydroxycyclohexanone, and cyclohexandione.

${ }^{\mathrm{d}}$ Filtered catalyst was reused in this batch.

${ }^{e}$ Oxidation of cyclooctene to epoxide in a $\mathrm{H}_{2} \mathrm{O}_{2} / t-\mathrm{BuOH}$ mixture. 
acid reached $30 \%$. For reaction time up to $30 \mathrm{~h}$ by adopting filtered WSBA15 catalyst, the selectivity toward adipic acid increased further to ca. 45\% [26]. Significantly, the TOFs reached 404 and 354 after 13 and $30 \mathrm{~h}$, respectively; these values are clearly superior to those reported previously for other inorganic catalysts, such as TiAPO-5 [13] and WMCM-41 [16]. HRTEM images clearly indicate that the long-range order of WSBA-15 was completely preserved even after multiple cycles of catalysis (Fig. 2c); these images reveal lattice mesophases of the cross-section parallel to the [001] direction and a corresponding electron diffraction pattern that are consistent with the 2D hexagonal mesostructure mentioned above. For determining tungsten oxide clusters bonded on silica framework after hydrothermal reaction, the energy dispersive X-ray (EDX) analysis is carried out. About $6 \mathrm{~mL}$ of filtered mixture solution heated at $80^{\circ} \mathrm{C}$ over $120 \mathrm{~h}$ was concentrated to less than $0.5 \mathrm{~mL}$. And $1 \mu \mathrm{L}$ of the solution was adapted for EDX analysis. Result shows the solution contained small amount of silicon elements without tungsten atoms. That means WSBA materials possess highly thermal stability and tungsten clusters are strongly bonded on the silica framework. Our results imply that, when bound to silica through $\mathrm{W}-\mathrm{O}-\mathrm{Si}$ covalent bonds, the isolated mixed-valence oxotungsten $\left\{\mathrm{WO}_{4}\right\}$ species act as catalytic centers for the oxidation of alkenes and possess the ability to release nascent oxygen gas formed from $\mathrm{H}_{2} \mathrm{O}_{2}$.

\section{Conclusions}

In conclusion, we describe in this paper a direct, environmentally benign method-involving aqueous $\mathrm{H}_{2} \mathrm{O}_{2}$ and a mesoporous catalyst based on WSBA-15, which greatly facilitates recycling and requires no organic solvent-for the direct oxidation of cyclohexene to colorless, crystalline adipic acid. We believe that the homogeneous distribution of tetrahedral oxotungsten entities within the channel walls is vital for the success of this catalytic process. We are undertaking further work toward providing an understanding of the intermediates that form and the mechanism of the overall reaction over this WSAB-15 catalyst.

\section{Acknowledgement}

This study was supported by the National Science Council of Taiwan (NSC-93-2113-M005-019; NSC-932120-M007-002).

\section{References}

[1] C.T. Kresge, M.E. Leonowlcz, W.J. Roth, J.C. Vartull, J.S. Beck, Nature 359 (1992) 710.

[2] J.S. Beck, J.C. Vartuli, W.J. Roth, M.E. Leonowicz, C.T. Kresge, K.D. Schmitt, C.T.-W. Chu, D.H. Olson, E.W. Sheppard, S.B. McCullen, J.B. Higgins, J.L. Schlenker, J. Am. Chem. Soc. 114 (1992) 10834.

[3] P.T. Tanev, T.J. Pinnavaia, Science 267 (1995) 865.

[4] P.T. Tanev, M. Chibwe, T.J. Pinnavaia, Nature 368 (1994) 321.

[5] D. Zhao, J. Feng, Q. Huo, N. Melosh, G.H. Fredrickson, B.F Chmelka, G.D. Stucky, Science 279 (1998) 548.

[6] D. Zhao, Q. Huo, J. Feng, B.F. Chmelka, G.D. Stucky, J. Am. Chem. Soc. 120 (1998) 6024.

[7] L. Nicole, C. Boissière, D. Grosso, P. Hesemann, J. Moreaub, C. Sanchez, Chem. Commun. (2004) 2312.

[8] T. Asefa, M.J. MacLachlan, N. Coombs, G.A. Ozin, Nature 402 (1999) 867.

[9] H. Fan, K. Yang, D.M. Boye, T. Sigmon, K.J. Malloy, H. Xu, G.P. López, C.J. Brinker, Science 304 (2004) 567.

[10] X.L. Yang, W.L. Dai, H. Chen, Y. Cao, H. Li, H. He, K. Fan, J. Catal. 229 (2005) 259.

[11] I.W.C.E. Arends, R.A. Sheldon, M. Wallau, U. Schuchardt, Angew. Chem., Int. Ed. 36 (1997) 1144.

[12] J.M. Thomas, R. Raja, G. Sankar, R.G. Bell, Nature 398 (1999) 227.

[13] S.O. Lee, R. Raja, K.D.M. Harris, J.M. Thomas, B.F.G. Johnson, G Sankar, Angew. Chem., Int. Ed. 42 (2003) 1520.

[14] G. Lapisardi, F. Chiker, F. Launay, J.P. Nogier, J.L. Bonardet, Catal. Commun. 5 (2004) 277.

[15] J.E. Herrera, J.H. Kwak, J.Z. Hu, Y. Wang, C.H.F. Peden, J. Macht, E. Iglesia, J. Catal. 239 (2006) 200.

[16] H. Chen, W.L. Dai, J.F. Deng, K. Fan, Catal. Lett. 81 (1-2) (2002) 131.

[17] K. Sato, M. Aoki, R. Noyori, Science 281 (1998) 1646.

[18] J.M. Brégeault, J.Y. Piquemal, E. Briot, E. Duprey, F. Launay, L. Salles, M. Vennat, A.P. Legrand, Micropor. Mesopor. Mater. 44-45 (2001) 409.

[19] E. Briot, J.Y. Piquemal, M. Vennat, J.M. Brégeault, G. Chottard, J.M. Manoli, J. Mater. Chem. 10 (2000) 953.

[20] Z. Zhang, J. Suo, X. Zhang, S. Li, Chem. Commun. (1998) 241.

[21] J. Jarupatrakorn, M.P. Coles, T.D. Tilley, Chem. Mater. 17 (2005) 1818.

[22] Bands at 300-390 $\mathrm{nm}$ can be assigned to octahedral species and bands at $390 \mathrm{~nm}$ to bulk $\mathrm{WO}_{3}$ See: M. Boulova, G. Lucazeau, J. Solid State Chem. 167 (2002) 425.

[23] The X-ray absorption near-edge spectral data for describing the ligand field geometry, electronic structure, and oxidation states of the metal centers will be published elsewhere.

[24] J. Wu, S.J. Li, J. Phys. Chem. 99 (1995) 4566.

[25] G. Ramis, C. Cristiani, A.S. Elmi, P. Villa, G. Busca, J. Mol. Catal. 61 (1990) 319.

[26] $\mathrm{O}_{2}$ - did not provide the conversion of adipic acid, but they did provide the yields of cyclohexanediol ( 27 and $24 \%$ for SAB- 15 and $\mathrm{H}_{2} \mathrm{O}_{2}$, respectively). 